\title{
Turbulent resistivity driven by the magnetorotational instability
}

\author{
S. Fromang ${ }^{1,2}$ and J. M. Stone ${ }^{3}$ \\ 1 CEA, Irfu, SAp, Centre de Saclay, 91191 Gif-sur-Yvette, France \\ e-mail: sebastien. fromang@cea.fr \\ 2 UMR AIM, CEA-CNRS-Univ. Paris VII, Centre de Saclay, 91191 Gif-sur-Yvette, France \\ 3 Department of Astrophysical Sciences, Peyton Hall, Ivy Lane, Princeton University, NJ 08544, USA
}

Received 23 June 2009 / Accepted 1 September 2009

ABSTRACT

\begin{abstract}
Aims. We measure the turbulent resistivity in the nonlinear regime of the MRI, and evaluate the turbulent magnetic Prandtl number. Methods. We perform a set of numerical simulations with the Eulerian finite volume codes Athena and Ramses in the framework of the shearing box model. We consider models including explicit dissipation coefficients and magnetic field topologies such that the net magnetic flux threading the box in both the vertical and azimuthal directions vanishes.

Results. We first demonstrate good agreement between the two codes by comparing the properties of the turbulent states in simulations having identical microscopic diffusion coefficients (viscosity and resistivity). We find the properties of the turbulence do not change when the box size is increased in the radial direction, provided it is elongated in the azimuthal direction. To measure the turbulent resistivity in the disk, we impose a fixed electromotive force on the flow and measure the amplitude of the saturated magnetic field that results. We obtain a turbulent resistivity that is in rough agreement with mean field theories like the Second Order Smoothing Approximation. The numerical value translates into a turbulent magnetic Prandtl number $P m_{\mathrm{t}}$ of order unity. $P m_{\mathrm{t}}$ appears to be an increasing function of the forcing we impose. It also becomes smaller as the box size is increased in the radial direction, in good agreement with previous results obtained in very large boxes.

Conclusions. Our results are in general agreement with other recently published papers studying the same problem but using different methodology. Thus, our conclusion that $P m_{\mathrm{t}}$ is of order unity appears robust.
\end{abstract}

Key words. accretion, accretion disks - magnetohydrodynamics (MHD) - methods: numerical

\section{Introduction}

Magnetic fields play a central role in accretion disk theory. Through the magnetorotational instability (MRI, Balbus \& Hawley 1991, 1998), they can explain why the flow is turbulent and how angular momentum is transported outward. They are also thought to be responsible for jet launching and collimation (Blandford \& Payne 1982; Pudritz et al. 2007). For both phenomena, whether or not a mean magnetic flux threads the disk has important consequences. Numerical simulations have indeed shown that the saturation level of the MRI depends strongly on the net flux: the famous $\alpha$ parameter (a measure of the rate of angular momentum transport through the disk, see Shakura \& Sunyaev 1973) is directly proportional to the square of the vertical magnetic field (Hawley et al. 1995). Similarly, a strong vertical field is mandatory for accretion - ejection models to be efficient at launching jets (Casse \& Ferreira 2000).

However, MHD turbulence not only can transport angular momentum outward in the disk, but also can diffuse magnetic flux radially. This possibility questions the very presence of a mean magnetic flux in the inner part of accretion disks (van Ballegooijen 1989; Lubow et al. 1994). Ultimately, how much magnetic flux resides in the inner disk depends on a competition between the effectiveness of outward angular momentum transport (i.e. how well mass and magnetic flux are advected inward) and magnetic flux diffusion. The former effect can be identified with a "turbulent viscosity". Similarly, the importance of the latter can be assessed through an equivalent "turbulent resistivity" $\eta_{\mathrm{t}}$ which has physical consequences similar to that of a microscopic resistivity. The purpose of this paper is to measure $\eta_{\mathrm{t}}$ accurately by using a set of local numerical simulations in which the flow is turbulent because of the MRI.

We note that two recently published papers have considered the same problem (Guan \& Gammie 2009; Lesur \& Longaretti 2009). Although all such studies (including our own) have used numerical simulations in the local shearing box model, there are significant differences between the approach used in each case. First, the numerical methods used to solve the MHD equations are different in all three approaches: Guan \& Gammie (2009) used numerical methods similar to those in the ZEUS code (Stone \& Norman 1992; Hawley \& Stone 1995) and Lesur \& Longaretti (2009) used a pseudo-spectral code in the incompressible limit. By contrast, we use two finite volume codes, Athena (Gardiner \& Stone 2005a, 2008; Stone et al. 2008) and Ramses (Teyssier 2002; Fromang et al. 2006). In addition, the method used in this paper to measure the turbulent resistivity (see Sect. 2) is different from that used by Guan \& Gammie (2009) and Lesur \& Longaretti (2009). The former add a large scale magnetic field to an already turbulent flow and associate the rate of decay of this field to the turbulent resistivity in the disk. The latter impose at all times an additional magnetic field in the computational domain, measure the time averaged electromotive force (EMF) associated with this field, and use the amplitude of the EMF to measure the turbulent resistivity. As described below, our approach is to impose a forcing EMF on the flow and relate the time averaged structure of the saturated field that results to the value $\eta_{\mathrm{t}}$. Another difference between our work and previous studies is that we study turbulence driven by a magnetic field with not net flux threading the box in any direction, while Guan \& Gammie (2009) and Lesur \& Longaretti (2009) 
perform numerical simulations in the presence of either a net vertical or a net toroidal field. It is well known that the presence of a net field affects the properties of the turbulence that develops (Hawley et al. 1995; Guan et al. 2009). Thus, an additional goal of this paper will be to study how much the final results obtained in all these studies depends on the details of the simulation setup. This will be useful to assess the robustness of the results.

The plan of this paper is as follows. In Sect. 2, we detail our method to measure the turbulent resistivity and the numerical setup we used. Section 3 serves essentially to validate our numerical codes by considering the case of a vanishing forcing EMF (in which case the simulations are standard shearing box simulations). We show that we obtain turbulent flows whose time- and volume-averaged properties are identical with both Athena and Ramses. We also study whether these results are modified when we consider boxes extended in the radial direction, as suggested recently in the literature (Johansen et al. 2009). These simulations serve as a basis for Sect. 4 in which we measure the turbulent resistivity in a number of situations, varying the strength and direction of the forcing EMFs, and the size of the box. Finally, in Sect. 5, we summarize our results, compare them with those of Guan \& Gammie (2009) and Lesur \& Longaretti (2009), and stress the limitations of our study.

\section{The setup}

\subsection{Equations}

We solve the equations of magnetohydrodynamics (MHD) in the framework of the shearing box model (Goldreich \& Lynden-Bell 1965) using two different codes: Athena (Gardiner \& Stone 2005a, 2008; Stone et al. 2008) and Ramses (Teyssier 2002; Fromang et al. 2006). Explicit microscopic dissipation, namely viscosity $v$ and resistivity $\eta$, are included in the simulations (note the microscopic resistivity $\eta$ should not be confused with the turbulent resistivity $\eta_{\mathrm{t}}$ that will be introduced below). It has been shown in the last few years that both are important in setting the saturated state of turbulence driven by the MRI (Lesur \& Longaretti 2007; Fromang et al. 2007). We use an isothermal equation of state $P=\rho c_{0}^{2}$ to relate density $\rho$ and pressure $P$, where $c_{0}$ is the sound speed. In a Cartesian coordinate system $(x, y, z)$ with unit vectors $(\boldsymbol{i}, \boldsymbol{j}, \boldsymbol{k})$ in the radial, azimuthal and vertical directions respectively, the equations for mass and momentum conservation are:

$$
\begin{aligned}
& \frac{\partial \rho}{\partial t}+\nabla \cdot(\rho \boldsymbol{v})=0, \\
& \frac{\partial \rho \boldsymbol{v}}{\partial t}+\boldsymbol{\nabla} \cdot(\rho \boldsymbol{v} \boldsymbol{v}-\boldsymbol{B} \boldsymbol{B})+\boldsymbol{\nabla} P_{t o t}=3 \rho \Omega x \boldsymbol{i}-2 \rho \boldsymbol{\Omega} \times \boldsymbol{v}+\nabla \cdot \boldsymbol{T}
\end{aligned}
$$

where $\Omega$ is the angular velocity of the shearing box around the central object, $\boldsymbol{B}$ is the magnetic field, $P_{\text {tot }}=P+(\boldsymbol{B} \cdot \boldsymbol{B}) / 2$ is the total pressure, and $\boldsymbol{T}$ is the viscous stress tensor (Landau \& Lifshitz 1959). The first two source terms on the right hand side of Eq. (2) represent the tidal gravity and Coriolis forces. Their implementation in Athena and Ramses is not straightforward. We followed the method described by Gardiner \& Stone (2005b) (see also Stone \& Gardiner 2009) and use a Crank-Nicholson algorithm to update the momentum fluctuations in time due to these source terms. The divergence of the viscous stress tensor in Eq. (2) is differenced in the conservative form so as to exactly conserve total momentum.
In order to study turbulent resistivity, we use a modified form of the induction equation:

$\frac{\partial \boldsymbol{B}}{\partial t}=\boldsymbol{\nabla} \times(\boldsymbol{v} \times \boldsymbol{B}-\eta \boldsymbol{\nabla} \times \boldsymbol{B}+\boldsymbol{E})$.

In this form we have introduced $\boldsymbol{E}$, an imposed electromotive force $(E M F)$, in addition to the usual inductive and resistive terms. When $\boldsymbol{E}=0$, as in Sect. 3, we recover the usual form of the induction equation for resistive MHD. In Sect. 4, we consider solutions to Eqs. (1)-(3) using the following expression for the imposed EMF:

$\boldsymbol{E}=E_{0 y} \cos \left(\frac{2 \pi x}{\lambda_{E, x}}\right) \boldsymbol{j}+E_{0 x} \cos \left(\frac{2 \pi z}{\lambda_{E, z}}\right) \boldsymbol{i}$,

where $\lambda_{E, x}$ and $\lambda_{E, z}$ are the radial and vertical wavelengths for the forcing EMF, respectively. To gain insight into the effect of $\boldsymbol{E}$, it is instructive to consider the case $v=0$. In this situation, the magnetic field reaches a steady state in which forcing due to $\boldsymbol{E}$ is balanced by resistive dissipation, with the field given by the following solution

$\boldsymbol{B}=-\frac{E_{0 x} \lambda_{E, z}}{2 \pi \eta} \sin \left(\frac{2 \pi z}{\lambda_{E, z}}\right) \boldsymbol{j}-\frac{E_{0 y} \lambda_{E, x}}{2 \pi \eta} \sin \left(\frac{2 \pi x}{\lambda_{E, x}}\right) \boldsymbol{k}$.

Equation (5) shows that the effect of the radial $(x)$ component of the EMF is to create a vertically varying azimuthal magnetic field, while the effect of the azimuthal $(y)$ component of the EMF is to create a radially varying vertical magnetic field. The amplitude of the field is determined by the amplitudes and wavelengths of the forcing and the resistivity. In a turbulent flow we expect the same balance will be achieved: turbulent velocity fluctuations will diffuse and reconnect the magnetic field that the driving EMF is trying to build. By analogy with Eq. (5), in a turbulent flow, we can define a turbulent resistivity $\eta_{\mathrm{t}}$ by measuring the amplitude of the spatially varying field resulting from the forcing. More specifically, we will consider two cases. First, $E_{0 x}=0$ and $E_{0 x}>0$, in which case the forcing results in a purely vertical field of amplitude $B_{z}^{0}$. This allows us to measure the turbulent resistivity $\eta_{\mathrm{t}, x}$ in the radial direction through the following relation, obtained using Eq. (5),

$\eta_{\mathrm{t}, x}=\frac{E_{0 y}}{2 \pi} \frac{\lambda_{E, x}}{B_{z}^{0}}$

Similarly, the case $E_{0 x}>0$ and $E_{0 x}=0$ yields a purely azimuthal field varying with $z$ and with an amplitude $B_{y}^{0}$ that measures the turbulent resistivity $\eta_{\mathrm{t}, z}$ in the vertical direction according to

$\eta_{\mathrm{t}, z}=\frac{E_{0 x}}{2 \pi} \frac{\lambda_{E, z}}{B_{y}^{0}}$

\subsection{Numerical method}

We solved Eqs. (1)-(3) using Athena and Ramses in a box of size $\left(L_{x}, L_{y}, L_{z}\right)=(H, 4 H, H)$, where $H=c_{0} / \Omega$ defines the vertical scale height (thickness) of the disk. We also used Athena to compute a few runs with a larger extent in the radial direction: $\left(L_{x}, L_{y}, L_{z}\right)=(4 H, 4 H, H)$. It has been shown recently by Johnson et al. (2008) that truncation error in the shearing box can create numerical artifacts, such as a density minima at the center of the box (where the shear velocity with respect to the grid is zero). We have implemented an orbital advection algorithm (Masset 2000; Gammie 2001; Johnson \& Gammie 2005; 
Johnson et al. 2008) in Athena (Stone \& Gardiner 2009) to alleviate this problem, and at the same time to allow for much larger timesteps in wide radial boxes.

All of our simulations are performed with no net magnetic flux in either the vertical or azimuthal direction. At $t=0$, we start with a purely vertical magnetic field varying sinusoidally with $x$ so that the mean field vanishes. Random velocity perturbations of small amplitude are applied to the background state, consisting of a uniform density gas with a linear shear profile: $\boldsymbol{v}=(0,-3 \Omega x / 2,0)$. We adopt $\Omega=c_{0}=10^{-3}$. In the rest of this paper, we measure time in units of the orbital period $T_{\text {orb }}=2 \pi / \Omega$. In all of the simulations we present below, we set $v$ and $\eta$ such that the Reynolds number $\operatorname{Re}=c_{0} H / \eta=3125$ and the magnetic Prandtl number $P m=4$. These coefficients are identical to the run labeled 128Re3125Pm4 of Fromang et al. (2007), and are known to lead to sustained turbulence over large numbers of orbital times. For explicit dissipation to dominate over numerical dissipation, we used a resolution of 128 grid points per $H$, or $(N x, N y, N z)=(128,512,128)$ for calculations that span one scale height in the radial direction. Such a resolution has been shown to give resolved solutions when using 2nd order Eulerian codes like ZEUS (Fromang et al. 2007) for microscopic dissipation coefficients of the magnitude adopted here. Simon et al. (2009) have recently shown that the numerical dissipation in Athena, likely comparable to Ramses, is similar in magnitude to that of ZEUS when performing numerical simulations of MRI - induced MHD turbulence in the shearing box. Thus, we expect the same resolution needed with ZEUS is also appropriate for the runs presented here that use Athena or Ramses. In simulations with larger boxes, we scaled the resolution accordingly, $(N x, N y, N z)=(512,512,128)$, so that the cell size remains the same.

Regardless of the code we use or the size of the computational domain, to study the turbulent resistivity induced by the MRI our strategy is as follows: we first performed a run without forcing (i.e. $\boldsymbol{E}=0$ ) to let the MRI develop and for turbulence to reach a steady state. Typically these runs are evolved for 100 orbits. They also serve as a useful comparison between codes, and to compare small vs. large boxes. At $t=30$, we restarted the simulations with forcing of various amplitudes imposed, as described above. With forcing, the flow evolves toward a new quasi steady - state that we use to evaluate the turbulent resistivity induced by the MRI. Before moving on to a description of the results with forcing in Sect. 4, we first describe the properties of MHD turbulence as obtained in runs without forcing in the next section.

\section{Runs without forcing}

The parameters of the runs we present in this section, along with time averaged values from selected quantities measured from the runs, are given in Table 1. Each run is labeled according to the code used ("R" for Ramses, or "A" for Athena) and the size of the box: "SB" for Small Box, i.e. simulations performed with $(L x, L y, L z)=(H, 4 H, H)$ and "LB" for Large Box, i.e. $(L x, L y, L z)=(4 H, 4 H, H)$. The resolution and duration of the runs are given in Cols. 3 and 4, respectively. In Col. 5, we report for each model the value of $\alpha$ time averaged between $t=50$ and the end of the simulation. As is usual, it is defined by the sum of the Reynolds and Maxwell stress tensors, $T_{r \phi}^{\text {Rey }}$ and $T_{r \phi}^{\mathrm{Max}}$, normalized by the volume averaged thermal pressure $\langle P\rangle$ :

$\alpha=\frac{T_{r \phi}^{\mathrm{Rey}}+T_{r \phi}^{\mathrm{Max}}}{\langle P\rangle}=\frac{\left\langle\rho\left(v_{x}-\overline{v_{x}}\right)\left(v_{y}-\overline{v_{y}}\right)\right\rangle-\left\langle B_{x} B_{y}\right\rangle}{\langle P\rangle}$, where $\overline{v_{x}}$ and $\overline{v_{y}}$ are $\mathrm{y}$ and $\mathrm{z}$ averages of $v_{x}$ and $v_{y}$ respectively, and \langle\rangle denotes a volume average. Finally, the last three columns in Table 1 give the amplitude of the gas velocity fluctuations along the three spatial coordinates.

We performed three runs. Two of them, R-SB and A-SB, share identical parameters but use different codes, Ramses and Athena respectively, in order to compare the results from both codes. The third one, A-LB, was performed with Athena in a larger domain. The last four columns of Table 1 show that the saturated state of the turbulence is almost identical in the three models. For example, $\alpha=0.014,0.015$ and 0.014 respectively in model R-SB, A-SB and A-LB. This agreement is confirmed by the three panels of Fig. 1 which correspond, from left to right, to $\mathrm{R}-\mathrm{SB}, \mathrm{A}-\mathrm{SB}$ and $\mathrm{A}-\mathrm{LB}$. All show the time history of $T_{r \phi}^{\mathrm{Rey}} /\langle P\rangle$ (lower dashed line), $T_{r \phi}^{\mathrm{Max}} /\langle P\rangle$ (upper dashed line) and $\alpha$ (solid line). The curves obtained in the three simulations are very similar and confirm the time averaged $\alpha$ values given in Table 1. These results have two implications. First, the good agreement between R-SB and A-SB gives confidence in both codes, as the two models have identical parameters and differ only in the algorithms. Thus, it validates both the implementation of the shearing box model in Ramses and the implementation of orbital advection in Athena. Given the rms fluctuations in $\alpha$ reported in Table 1, these results are also consistent with the value of $\alpha \sim 0.01$ quoted by Fromang et al. (2007) for their model 128Re3125Pm4. We note, nonetheless, that the results obtained here with Athena and Ramses appear to lead to slightly larger value of the angular momentum transport. It is unclear (and beyond the scope of this paper) whether this difference is significant. It may be partly due to our box size being slightly larger in the azimuthal direction compared to that used in Fromang et al. (2007), or it could be due to small differences in the numerical dissipation between the various codes. The second result that emerges from the models presented in this section is the insensitivity of the turbulence properties to the box size. The time averaged value of $\alpha$ in model A-LB is almost identical to the other two. The time history presented on the right panel of Fig. 1 also shows good agreement with the other two panels. The only difference is that the fluctuations around the mean $\alpha$ value are smaller in the case of the big box. This translates into a standard deviation which is about three times smaller in A-LB than in the other two models. Again, the reasons for this difference are unclear. It may be due to better statistics in the large box (simply due to the larger volume of the simulations) that average over extreme events. Alternatively, it might be due to the larger number of parasitic modes than can develop in larger boxes (Pessah \& Goodman 2009) or to a more efficient nonlinear coupling between the more numerous turbulent modes present in a larger box (Latter et al. 2009). Both effects would reduce the lifetime (and therefore the influence) of channel modes. The similarity between the small and large boxes model is further confirmed by Figs. 2 and 3. The former shows snapshots of the density (left panel), $B_{y}$ (middle panel) and $v_{z}$ (right panel) in the $(x, z)$ plane for model R-SB at $t=70$ (very similar figures are obtained for model A-SB). The equivalent figures for model A-LB at $t=70$ are shown in Fig. 3. The figure shows that the structure of the flow in the large box is very similar to four patches of the small box model repeated next to each other.

Other features typical of shearing box simulations of the MRI, like density wave propagating radially in the box (Heinemann \& Papaloizou 2008a,b), are clearly seen in the density field regardless of the box size. Finally, as expected for a simulation having $P m>1$, the smallest scale structure seen in 
Table 1. Properties of MHD turbulence when forcing is turned off.

\begin{tabular}{cccccccc}
\hline \hline Model & Box size & Resolution & $t_{\text {lim }}$ & $\alpha$ & $\delta v_{x} / c_{0}$ & $\delta v_{y} / c_{0}$ & $\delta v_{z} / c_{0}$ \\
\hline R-SB & $(H, 4 H, H)$ & $(128,512,128)$ & 160 & $1.4 \times 10^{-2} \pm 3.6 \times 10^{-3}$ & 0.0982 & 0.0776 & 0.0609 \\
A-SB & $(H, 4 H, H)$ & $(128,512,128)$ & 100 & $1.5 \times 10^{-2} \pm 5.7 \times 10^{-3}$ & 0.0936 & 0.0774 & 0.0588 \\
A-LB & $(4 H, 4 H, H)$ & $(512,512,128)$ & 100 & $1.4 \times 10^{-2} \pm 1.4 \times 10^{-3}$ & 0.0945 & 0.0815 & 0.0573 \\
\hline
\end{tabular}

The first column labels the model. The box size, resolution and duration (in orbits) of the simulations are given in columns two, three and four. The time averaged properties of the turbulence, i.e. the value of $\alpha$ (total stress normalized by the thermal pressure) and the velocity fluctuations along the three spatial (normalized by the speed of sound) appear in the following four columns.
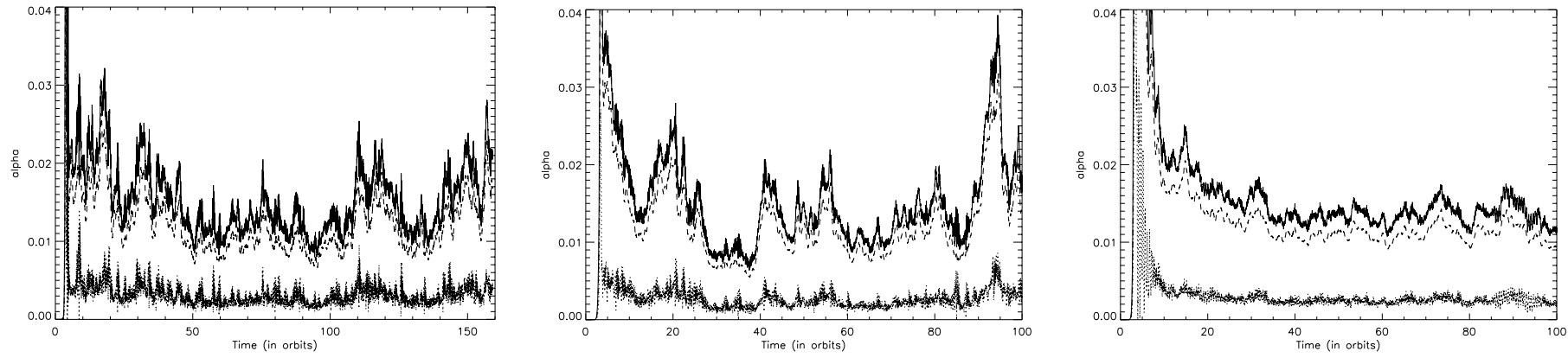

Fig. 1. Time history of $\alpha$ (solid line), the Maxwell stress $T_{r \phi}^{\mathrm{Max}}$ (dashed line) and the Reynolds stress $T_{r \phi}^{\mathrm{Rey}}$ (dotted line). From left to right, the three panels show the results of model $R-S B, A-S B$ and $A-L B$ respectively. For all cases, $R e=3125$ and $P m=4$.
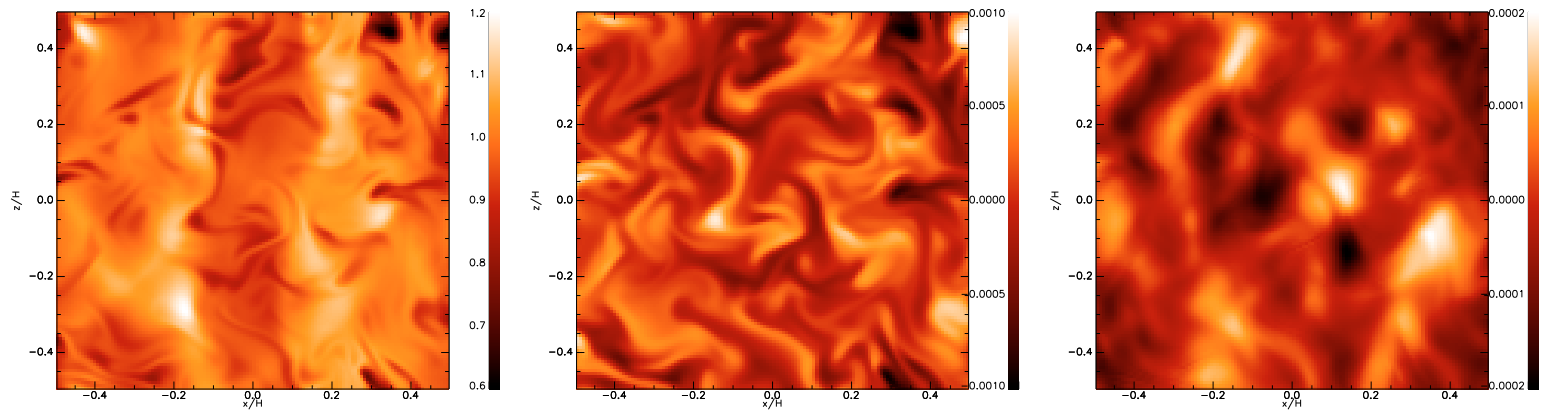

Fig. 2. Structure of the flow in the $(x, z)$ plane obtained in model $R-S B$ at $t=70$ orbits. The left, middle and right panels show the density, azimuthal component of the magnetic field, and vertical component of the velocity respectively. Similar results are obtained in model $A-S B$.

the velocity field is generally larger than the smallest scale structure seen in the magnetic field. The latter shows elongated filaments reminiscent of high $\mathrm{Pm}$ small scale dynamo simulations (see for example Schekochihin et al. 2007).

Recently, Johansen et al. (2009) also reported numerical simulations of MHD turbulence in the shearing box in large domains. They found an increase of $\alpha$ when going from small $(H)$ to large $(4 H)$ boxes. Their result differs from those reported here, where we find no sensitivity of $\alpha$ on box size. The reason probably lies in the fact that we always use domains with an azimuthal extend of $4 H$, even when the radial domain is small (i.e. when $L_{x}=H$ ). On the other hand, Johansen et al. (2009) used square domains, with $L_{x}=L_{y}=H$ in their small box. It is well known that the azimuthal extent of the domain affects the saturated level of the turbulence (Hawley et al. 1995, 1996). We conclude that, for fixed and large azimuthal extent of the domain, the converged value of $\alpha$ is insensitive to the radial dimension of the box, at least for the values of the Reynolds and Prandtl numbers studied here.

Another property of the flow reported by Johansen et al. (2009) is the existence of large scale, long-lived zonal flows that generate axisymmetric density features in large boxes. We have looked for, and found, such features in our large box simulations as well. An example is shown in Fig. 4, a space-time diagram of the azimuthally and vertically averaged density in model A-LB. It is similar to Fig. 6 of Johansen et al. (2009) and shows a similar amplitude and lifetime for large scale density features, indicating that a zonal flows also develops in our simulations. As both results were obtained using completely different codes, our results confirm the conclusion drawn by Johansen et al. (2009): zonal flows appear to be a robust feature in MRIdriven turbulence in the shearing box.

This completes our description of the models computed in the absence of a forcing EMF. The flow in these models provides a starting point to study turbulent resistivity induced by the MRI. Namely, at $t=30$, we restarted the models using various amplitudes and directions for the forcing EMF, $\boldsymbol{E}$. The following section describe these results.

\section{Turbulent resistivity}

The properties and results of the runs we performed are listed in Table 2. The first column gives a label for the name, coded according to the following convention: "direction-amplitude-codeparameters" where "direction" can be either "Ey" or "Ex" and indicates the direction of the forcing EMF (we considered only forcing aligned along either the radial or the azimuthal direction, respectively creating vertical or toroidal field), "amplitude" 

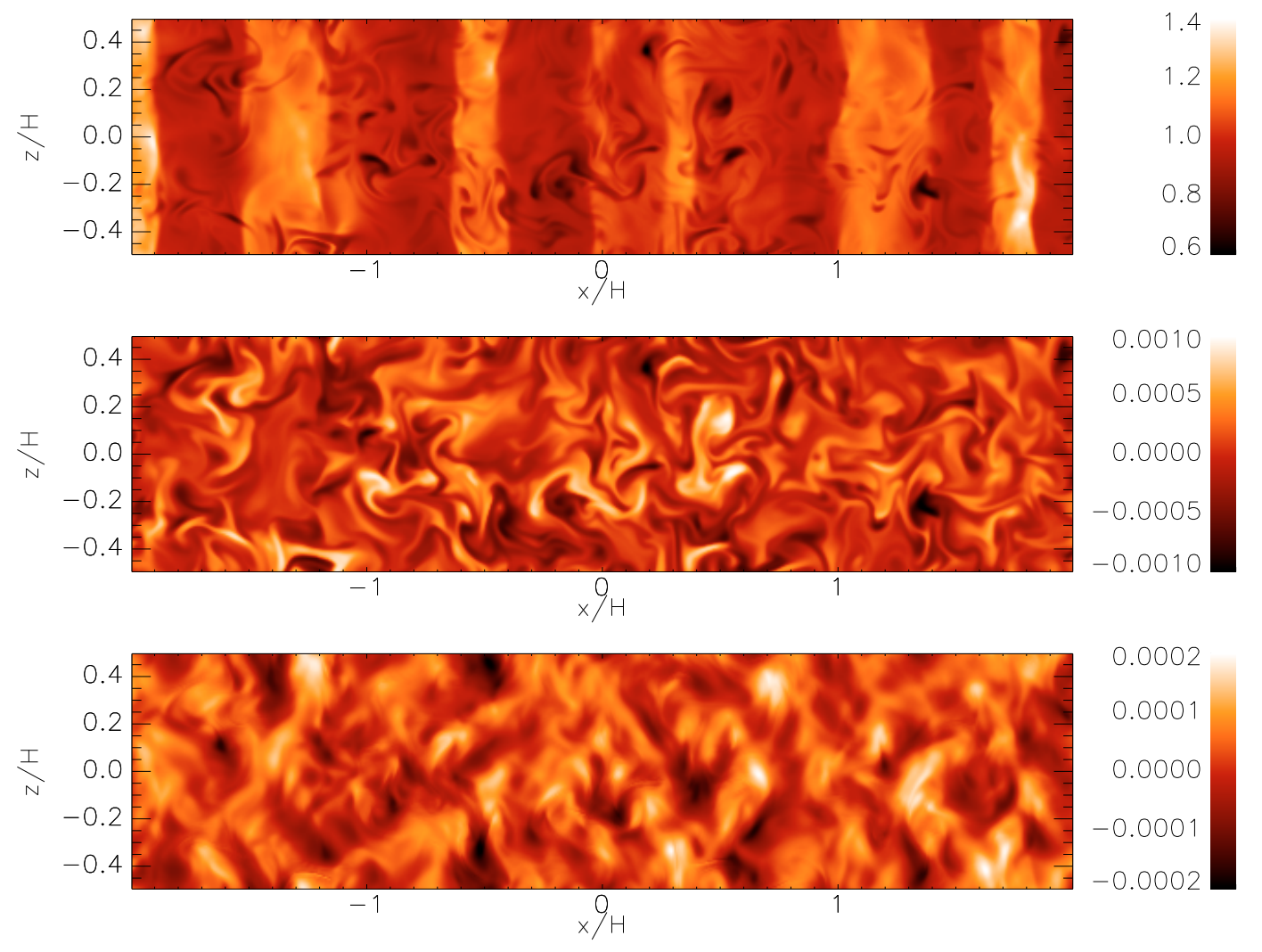

Fig. 3. Same as Fig. 2 but for model $A-L B$ : the upper, middle and lower panels show the density, azimuthal component of the magnetic field, and vertical component of the velocity respectively.

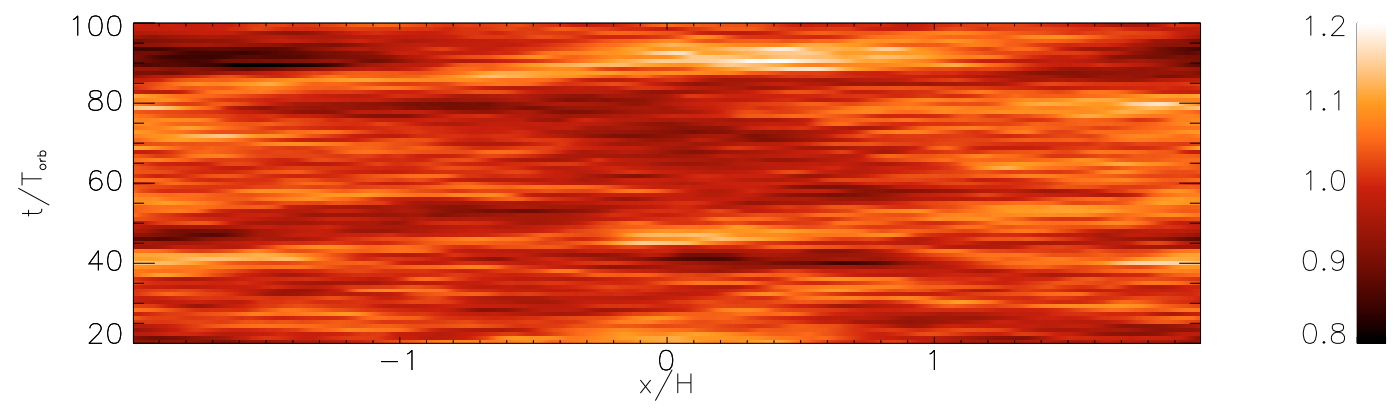

Fig. 4. Space-time diagram showing the radial variation of the density, averaged along $y$ and $z$, in model $A-L B$. It shows the appearance of long-lived density features. They are associated with large scale zonal flows as recently reported by Johansen et al. (2009).

gives the amplitude of $\boldsymbol{E}$, "code" reports the code we used for that model ("R" for Ramses, and "A" for Athena), while "parameters", whenever present, gives additional parameters that will be specified when needed in the text. For example, model $E y-4 E$ $10-R$ was performed with Ramses using a forcing EMF in the azimuthal direction of amplitude $4 \times 10^{-10}$. The other parameters reported in Table 2 are the duration over which the data were averaged (Col. 2), the amplitude of the forcing EMF (Col. 3), the value of $\alpha$ (Col. 4) and the amplitude of the velocity fluctuations along the three spatial coordinates (Cols. 5-7).

The forcing EMF we imposed on the flow results in a steadystate magnetic field profile. We fitted the profile (averaged in time over the duration of the run and in space over the two directions perpendicular to the direction of variation) by a sinusoidal function. The amplitude of the fit, $B_{\text {fit }}$, is expressed via the parameter

$\beta_{\max }=\frac{\langle P\rangle}{B_{\mathrm{fit}}^{2} / 2}$, and given in Col. 8 on Table 2 . The turbulent resistivity $\eta_{\mathrm{t}}$ responsible for that amplitude is calculated using Eqs. (6) or (7), depending of the direction of the forcing EMF. Its value is reported in Col. 9.

Finally, we follow Guan \& Gammie (2009) and associate a turbulent "viscosity" $v_{\mathrm{t}}$ with the flow, where

$v_{\mathrm{t}}=\frac{2}{3} \alpha c_{0} H$

which we use to define a turbulent magnetic Prandtl number $P m_{\mathrm{t}}=v_{\mathrm{t}} / \eta_{\mathrm{t}}$ given in Col. 10 .

\subsection{Radial diffusion of a vertical magnetic field}

4.1.1. $E_{\text {Oy }}=4 \times 10^{-10}$

In this section, we describe in detail the results we obtained for models Ey-4E-10-R, Ey-4E-10-R-LONG and Ey-4E-10-A. All 
Table 2. Properties of MHD turbulence when a forcing EMF is turned on.

\begin{tabular}{|c|c|c|c|c|c|c|c|c|c|}
\hline$\overline{\overline{\text { Model }}}$ & 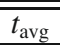 & $\overline{|\bar{E}|}$ & 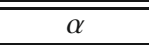 & 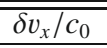 & 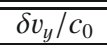 & $\overline{\delta \delta v_{z} / c_{0}}$ & 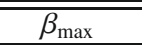 & $\overline{\overline{\eta_{\mathrm{t}}}}$ & $P P m_{\mathrm{t}}$ \\
\hline $\begin{array}{l}\text { Ey-4E-10-R } \\
\text { - }\end{array}$ & 50 & $4 \times 10^{-10}$ & $2.3 \times 10^{-2}$ & 0.1214 & 0.1060 & 0.0789 & $5.6 \times 10^{4}$ & $1.07 \times 10^{-5}$ & 1.47 \\
\hline Ey-4E-10-R-LONG & 120 & $4 \times 10^{-10}$ & $2.0 \times 10^{-2}$ & 0.1173 & 0.1001 & 0.0758 & $5.5 \times 10^{4}$ & $1.06 \times 10^{-5}$ & 1.27 \\
\hline Ey-1E-9-R & 50 & $1 \times 10^{-9}$ & $2.6 \times 10^{-2}$ & 0.1344 & 0.1231 & 0.0896 & $1.7 \times 10^{4}$ & $1.46 \times 10^{-5}$ & 1.21 \\
\hline Ey-4E-9-R & 50 & $4 \times 10^{-9}$ & $5.2 \times 10^{-2}$ & 0.1831 & 0.1885 & 0.1246 & $1.6 \times 10^{3}$ & $1.81 \times 10^{-5}$ & 1.91 \\
\hline Ey-8E-9-R & 50 & $8 \times 10^{-9}$ & $7.6 \times 10^{-2}$ & 0.2209 & 0.2458 & 0.1493 & $7.3 \times 10^{2}$ & $2.43 \times 10^{-5}$ & 2.10 \\
\hline Ey-4E-10-A & 50 & $4 \times 10^{-10}$ & $1.7 \times 10^{-2}$ & 0.1020 & 0.0846 & 0.0633 & $5.8 \times 10^{4}$ & $1.08 \times 10^{-5}$ & 1.02 \\
\hline Ey-4E-9-A & 50 & $4 \times 10^{-9}$ & $5.3 \times 10^{-2}$ & 0.1766 & 0.1876 & 0.1189 & $1.2 \times 10^{3}$ & $1.56 \times 10^{-5}$ & 2.28 \\
\hline Ey-4E-10-A-L4-1x4 & 48 & $4 \times 10^{-10}$ & $2.6 \times 10^{-2}$ & 0.1242 & 0.1202 & 0.0798 & $2.8 \times 10^{4}$ & $3.02 \times 10^{-5}$ & 0.57 \\
\hline Ey-4E-9-A-L4-1x4 & 50 & $4 \times 10^{-9}$ & $1.0 \times 10^{-1}$ & 0.2371 & 0.2624 & 0.1555 & $1.4 \times 10^{3}$ & $6.68 \times 10^{-5}$ & 1.00 \\
\hline Ey-4E-9-A-L4-1x1 & 50 & $4 \times 10^{-9}$ & $5.3 \times 10^{-2}$ & 0.1789 & 0.1875 & 0.1204 & $1.6 \times 10^{3}$ & $1.82 \times 10^{-5}$ & 1.93 \\
\hline$\overline{\text { Ex-4E-9-R }}$ & 50 & $4 \times 10^{-9}$ & $2.4 \times 10^{-2}$ & 0.1292 & 0.1162 & 0.08476 & $5.3 \times 10^{2}$ & $1.05 \times 10^{-5}$ & 1.57 \\
\hline Ex-8E-9-R & 50 & $8 \times 10^{-9}$ & $4.6 \times 10^{-2}$ & 0.1746 & 0.1747 & 0.1160 & $1.3 \times 10^{2}$ & $1.01 \times 10^{-5}$ & 3.00 \\
\hline
\end{tabular}

The first column gives the model label, the second the duration over which the data are averaged, and the third the amplitude of the forcing EMF used in that case. Subsequent columns give time averaged values of various quantities of physical interest, namely the mean values of $\alpha$, the maximum value of $\beta$ (ratio between the thermal and magnetic pressure), the value of the turbulent resistivity that results from fitting the mean vertical magnetic field, and the turbulent magnetic Prandtl number of the flow.
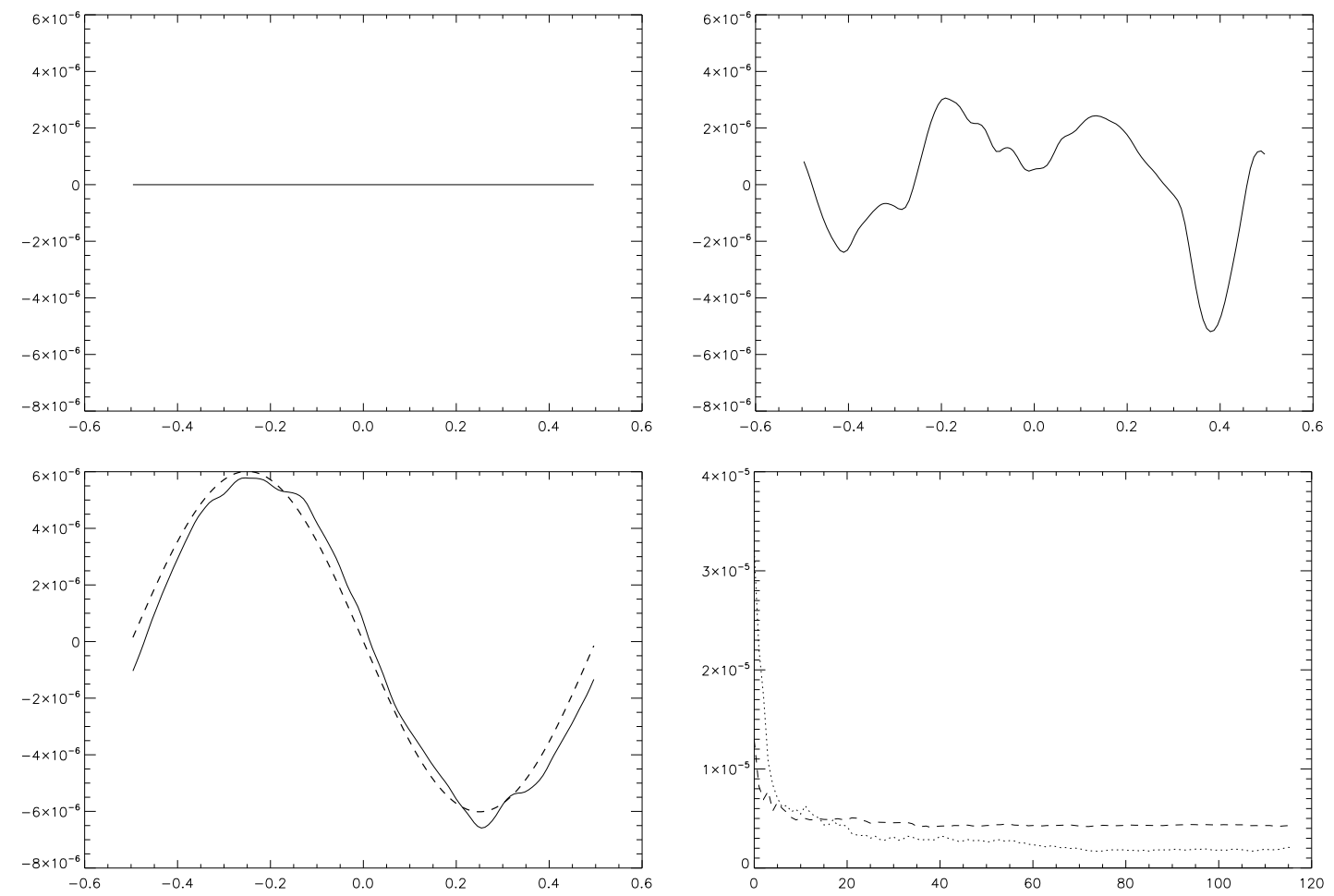

Fig. 5. Time averaged radial profile of the vertically and azimuthally averaged radial (upper left panel), azimuthal (upper right panel) and vertical (lower left panel) magnetic field for model Ey-4E-10-R-LONG. On the third panel, the solid line shows the curve extracted from the numerical simulations while the dashed line is a least-squared fit to the data assuming a sinusoidal profile. The lower right panel shows the time variation of the standard deviation of the three magnetic field components, plotted using solid, dotted and dashed lines respectively. These plots illustrate that the forcing EMF creates, as expected, a radially varying vertical field with an amplitude larger than the fluctuations of the other magnetic field components.

were obtained using a forcing EMF directed along the azimuthal direction, and varying only in the radial direction: $E_{0 x}=0$ and $E_{0 y}=4 \times 10^{-10}$. We used $\lambda_{E, x}=H$. The first two were computed using Ramses and differ only in the duration of the averaging procedure we applied. The third was computed using Athena and serves both to validate our method and to quantify the uncertainty in our estimate of the turbulent resistivity and magnetic Prandtl number.
The results we obtained for this set of parameters are illustrated in Fig. 5 for model Ey-4E-10-R-LONG. There are four panels. The solid line in the first three (upper left, upper right and lower left) plots the radial profile of $B_{x}, B_{y}$ and $B_{z}$ respectively, averaged in time between $t=30$ and $t=150$ using 120 dumps, and averaged in space over $y$ and $z$. All plots use the same vertical scale. It is apparent from the lower left panel that the toroidal forcing EMF created a sinusoidally varying vertical field, as 
expected from the discussion in Sect. 2. The dashed line shows the sinusoidal curve obtained by a least square fit to the data, and gives an amplitude $B_{0 z} \sim 6 \times 10^{-6}$ (this translates into a value $\beta_{\max }=5.5 \times 10^{4}$ as shown in Table 2 ). It is immediately obvious than this rather low value is mostly due to the effect of turbulence. Indeed, using Eq. (5), the effect of the microscopic resistivity alone would give an amplitude $B_{0 z}^{\eta} \sim 8 \times 10^{-4}$, larger by about two orders of magnitude than the measured value of the vertical field. Using Eq. (6), we converted $B_{0 z}$ into a turbulent resistivity $\eta_{\text {turb }, x}=1.06 \times 10^{-5}$. Combined with the time averaged value of $\alpha=2.0 \times 10^{-3}$, this gives a turbulent magnetic Prandtl number of $P m_{\mathrm{t}}=1.27$.

Given the rather low value of the forcing EMF, it is reasonable to question the accuracy of these measurements. Indeed, the second and third panels in Fig. 5 indicate that the fluctuations in $B_{y}$ (upper right panel) are of comparable amplitude to $B_{0 z}{ }^{1}$. The fourth (lower right) panel, which shows the time history of the rms fluctuations in the running time and spatial averages of $B_{z}$ (solid line) and $B_{y}$ (dashed line), indicates a similar trend: although the rms fluctuations of $B_{z}$ become larger than those of $B_{y}$ after about 20 orbits (thus indicating than the standard averaging duration of 50 orbits we usually take in the following is enough), the ratio between the two is only a factor of about two after 120 orbits. In other words, the vertical field generated by the forcing EMF is not much larger than the turbulent fluctuations in the field. As such, our measurements are susceptible to a rather large uncertainty ${ }^{2}$. In order to quantify this uncertainty, we consider model $E y-4 E-10-R$, which is the same as model $E y-4 E-10-R-L O N G$ but averaged over only 50 orbits, and model $E y-4 E-10-A$, identical to model $E y-4 E-10-R$ but performed using Athena. As shown on Table 2, we found extremely close values for the turbulent resistivities for all three models. The measured turbulent magnetic Prandtl number varies by about $50 \%$, from $P m_{\mathrm{t}} \sim 1$ to $P m_{\mathrm{t}} \sim 1.5$. This difference can immediately be attributed to variations in the measured $\alpha$, ranging from $1.7 \times 10^{-3}$ to $2.3 \times 10^{-3}$. Such a variation is compatible with the standard deviation of $\alpha$ reported in Table 1 and we conclude therefore that a safe estimate of the turbulent Prandtl number in the radial direction in this case lies in the range [1., 1.5].

This estimate is in rough agreement with the recent results reported by Guan \& Gammie (2009) and Lesur \& Longaretti (2009) in simulations performed in the presence of a net magnetic field in the azimuthal or vertical direction. It is also reassuring that our measured value for the turbulent resistivity is in rough agreement with mean field theories like the SecondOrder Correlation Approximation (SOCA, Rädler \& Rheinhardt 2007) also known as the First Order Smoothing Approximation (FOSA, Brandenburg \& Subramanian 2005). In this approach, the turbulent resistivity is expected to take the form

$\eta_{\mathrm{t}, x}^{\mathrm{SOCA}}=\delta v_{x}^{2} \tau_{\mathrm{corr}}$,

where $\tau_{\text {corr }}$ is the correlation time of the turbulent velocity fluctuations. Estimates for $\tau_{\text {corr }}$ are not straightforward to obtain. In a set of local and global stratified simulations of MRI-induced turbulence having $\alpha \sim 0.01$, Fromang \& Papaloizou (2006) and

\footnotetext{
1 The absence of fluctuations in the $y$ and $z$ averaged radial profile of $B_{x}$ comes from the solenoidal nature of the magnetic field.

2 The reason to study such small fields is so that they do not significantly affect the property of the turbulence itself (the wavelength of the most unstable wavelength of the MRI associated with the field generated by the forcing EMF is largely underesolved in our simulations). Thus, they merely act as a passive probe of the turbulence, which is not the case for the larger amplitude of $\boldsymbol{E}$ we consider in Sect. 4.1.2.
}

Fromang $\&$ Nelson (2009) found $\tau_{\text {corr }} \sim 0.15 T_{\text {orb. Adopting this }}$ estimate here, and using $\delta v_{x} \sim 0.11 c_{0}$ given in Table 2 for the three models we consider in this section, Eq. (11) then gives

$\eta_{\mathrm{t}, x}^{\mathrm{SOCA}}=1.14 \times 10^{-5}$

a value that is, given the approximations involved in the SOCA, in very good agreement with the results reported in Table 2.

\subsubsection{Varying the forcing EMFs}

In the previous section, we considered a forcing whose amplitude only produces a very weak effect on the underlying turbulence. In this section, we relax this hypothesis by considering larger forcing EMFs. Such forcing may have an effect on the saturated properties of the turbulence itself, which in turn may change the values of the turbulent resistivity and $P m_{\mathrm{t}}$.

In the run we performed, listed in Table 2, the amplitude of the EMF was varied between $|\boldsymbol{E}|=4 \times 10^{-10}$ (described in the previous section) and $|\boldsymbol{E}|=8 \times 10^{-9}$, keeping $\lambda_{E, x}=H$ in all cases. We tried larger values as well but found that, regardless of the code we used, the flow developed regions of low density and large magnetic field. In these regions the large Alfven speed resulted in an extremely small timestep, which prevented the simulations from being continued.

Figure 6 shows the radial profile (time averaged for 50 orbits and spatially averaged over the azimuthal and vertical directions) of the vertical magnetic field we obtained in models $E y-1 E-9-R$ (left panel), Ey-4E-9-R (middle panel) and Ey-8E-9-R (right panel). As was the case for the lower left panel of Fig. 5, the solid line plots the results of the simulation and the dashed line shows the sinusoidal fit used to derive the turbulent resistivity and magnetic Prandtl number. As expected, Fig. 6 shows that the amplitude of the magnetic field increases with the forcing amplitude. It also illustrates the quality of the sinusoidal fit for these models. The turbulent resistivity and magnetic Prandtl numbers we calculate using the amplitude of the steady-state field are shown in Table 2. We also report (Cols. 4 to 7) the time averaged $\alpha$ values and time averaged velocity fluctuations measured in these runs. As forcing is increased, we find that $\eta_{t, x}$ increases. Increasing the forcing by a a factor of 20 increases $\eta_{t, x}$ by about 2.5 . At the same time, $\alpha$ roughly triples. This leads to a mild but systematic increase in $P m_{\mathrm{t}}$ with the amplitude of the forced vertical magnetic field. To further test our results, we also computed a model with $|\boldsymbol{E}|=4 \times 10^{-9}$ with Athena (model Ey-4E-9-A in Table 2). The results are seen to be consistent with the results obtained with Ramses.

Since Eq. (11) predicts the scaling of $\eta_{t, x}$ with the velocity fluctuations, in Fig. 7 we show our results in the $\left(\delta v_{x}, \eta_{t, x}\right)$ plane. The data obtained with Ramses are plotted using diamonds while the points computed using Athena appear as a star symbol. The dotted line is a naive estimate that uses the prediction of the SOCA given by Eq. (11), assuming that the correlation timescale is independent of the velocity fluctuations: $\tau_{\text {corr }}=0.15 T_{\text {orb }}$. Clearly, the dotted line does not fit the data. This is most likely because $\tau_{\text {corr }}$ varies as the strength of the turbulence changes. On dimensional grounds, it can be estimated that

$\tau_{\text {corr }} \sim l_{x, \text { corr }} / \delta v_{x}$

where $l_{x, \text { corr }}$ is a correlation length associated with the vertical magnetic field fluctuations. Although it is rather ill-defined, an 

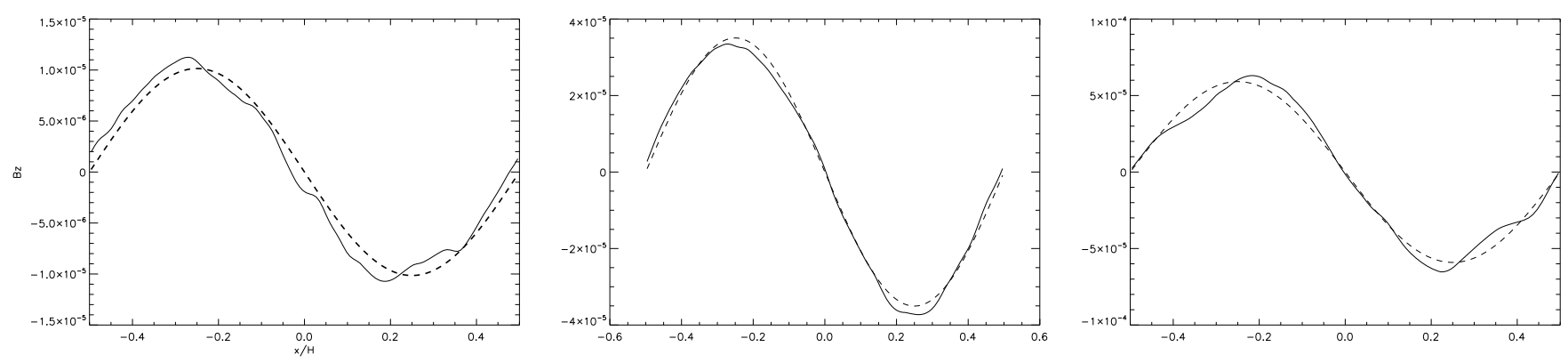

Fig. 6. Vertically, azimuthally and time averaged radial profile of the vertical magnetic field for models Ey-1E-9-R (left panel), Ey-4E-9-R (middle panel) and $E y-8 E-9-R$ (right panel). On each panel, the dashed line is a sinusoidal fit to the simulation data used to estimate the turbulent resistivities reported in Table 2.

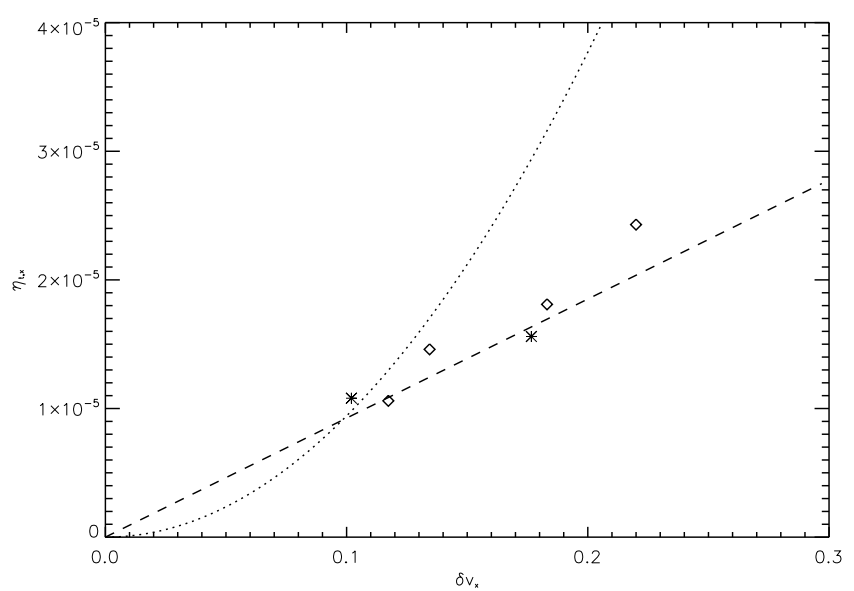

Fig. 7. Turbulent resistivity in the radial direction as a function of the turbulent velocity fluctuations for various models computed using different amplitude of the forcing EMFs. Diamonds correspond to run performed using Ramses while stars use the results of models computed with Athena. The dotted line shows the prediction of SOCA assuming a constant correlation time, independent of the velocity fluctuations, while the dashed line shows the same prediction but computed assuming a correlation lengthscale for the magnetic field independent of the amplitude of the velocity fluctuations (see text for details). The latter is seen to be in good agreement with the data.

order of magnitude estimate for $l_{x, \text { corr }}$ can be obtained as (Lesur \& Longaretti 2007)

$l_{x, \text { corr }} \sim\left\langle\frac{\int B_{z}(x, y, z) B_{z}\left(x^{\prime}, y, z\right) \mathrm{d} x \mathrm{~d} x^{\prime}}{\int B_{z}^{2}(x, y, z) \mathrm{d} x}\right\rangle$,

where angled brackets denote a spatial average over the $y$ and $z$ directions followed by a time average. Using Eq. (14), we found that $l_{x, \text { corr }}$ is only weakly varying with the strength of the turbulence. Indeed, we obtained $0.06 H<l_{x \text {,corr }}<0.07 H$ for forcing amplitudes ranging from $|\boldsymbol{E}|=0$ (i.e. no forcing) to $|\boldsymbol{E}|=8 \times 10^{-9}$ (i.e. the largest forcing amplitude we considered). It would be dangerous at this stage to take the estimate given by Eq. (14) as a good way of calculating $\tau_{\text {corr }}$, as Eq. (13) is only valid on dimensional grounds. Instead, we used the independence of $l_{x, \text { corr }}$ to write the correlation timescale as

$\tau_{\text {corr }} \sim \tau_{0} \frac{\delta v_{x 0}}{\delta v_{x}}$ where $\tau_{0}=0.15 T_{\text {orb }}$ and $\delta v_{x 0}$ denotes the velocity fluctuations in the absence of forcing (given in Table 1). This gives

$\eta_{\mathrm{t}, x}^{\mathrm{SOCA}}=\delta v_{x} \delta v_{x 0} \tau_{0}$,

which we used to compute the dashed line shown in Fig. 7. The agreement with the data is much better and captures the scaling of the turbulent resistivity as the amplitude of the forcing EMFs is varied by more than one order of magnitude.

\subsection{Radial diffusion of a vertical field in extended boxes}

Next, we consider the turbulent resistivity in boxes extended in the radial direction. These models are based on the results of model $A-L B$, which we restarted at time $t=30$ with forcing of different amplitudes. We only consider forcing in the azimuthal direction in this case. Again, we report the properties of the turbulence and the values of $\eta_{\mathrm{t}, x}$ and $P m_{\mathrm{t}}$ we obtained in Table 2.

To check the results obtained in smaller boxes, we first consider model Ey-4E-9-A-L4-lxl. In this case, we used $\lambda_{E, x}=H$ and $E_{0 y}=4 \times 10^{-10}$. Thus, it is the big box equivalent of models $E y-4 E-9-R$ and $E y-4 E-9-A$. We therefore expect it to yield the same results. Table 2 shows that this is indeed the case, with the values of $\alpha, \eta_{t, x}$ and $P m_{\mathrm{t}}$ being nearly the same in all three models.

Next, we turn to models Ey-4E-10-A-LA-lx4 and Ey-4E-9-A$L 4$-lx4 for which $\lambda_{E, x}=4 H$. The first result to note is that the amplitude of the vertical field we obtained is larger in these two cases than in the small box models with equal forcing EMFs. This was somewhat expected. Since $\lambda_{E, x}$ is now larger, the local gradient of the vertical field are smaller in the big boxes and the diffusion of magnetic field is less efficient. Nevertheless, the measured turbulent resistivity are significantly larger than their small boxes counterpart. At the same time, the velocity fluctuations in the radial direction are comparable to the small boxes models having the same forced vertical field amplitude. It is likely that all other statistical properties of the turbulence share the same insensitivity to box size, which means that a straightforward application of the SOCA breaks down in this case. The increased resistivity is therefore due to the increased wavelength $\lambda_{E, x}$ of the forcing EMF used in that case. One possible explanation, to be taken with care, for such large $\eta_{\mathrm{t}}$ can then be described as follows: the resulting forced magnetic field, having a larger scale, will most likely be diffused by larger eddies. Because the kinetic energy power spectrum is decreasing with wavenumber, such eddies have a larger kinetic energy that makes them more efficient at diffusing the magnetic field. 
The consequence of the larger turbulent resistivities obtained in our large box simulations is lower values of $P m_{\mathrm{t}}$. In the case of model Ey-4E-10-A-L4-lx4,Pm $\sim 0.5$, i.e. a factor of about three times smaller compared to the small boxes. We recover $P m_{\mathrm{t}}$ of order unity for model Ey-4E-9-A-L4-lx4 owing to a larger value of $\alpha$. This is presumably due to the fact that the flow starts to behave as if threaded by a net vertical flux for this long wavelength, large amplitude forcing.

\subsection{Vertical diffusion of an azimuthal magnetic field}

In addition to studying radial diffusion of vertical magnetic fields as described in the previous section, we also performed simulations in which we study the vertical diffusion of an azimuthal magnetic field. In this case, we restricted our analysis to small boxes, and we chose $E_{0 y}=0$ and therefore considered forcing EMFs purely in the radial direction. We allowed for variations of the amplitude of the forcing in the range $4 \times 10^{-9}<E_{0 x}<8 \times$ $10^{-9}$. We also tried $E_{0 x}=4 \times 10^{-10}$ as in the case of radial diffusion. However, because the azimuthal magnetic field fluctuations are of larger amplitudes than the vertical field fluctuations, it has proven difficult to extract a meaningful mean field from that model. We therefore decided to consider only large amplitudes for the forcing EMFs.

The results we obtained are summarized once more in Table 2. We obtain turbulent resisitivities that are generally lower than for the case of radial field diffusion. This is not inconsistent with the prediction of SOCA, as the velocity fluctuations in the vertical direction are of lower amplitudes than the velocity fluctuations in the radial direction. Using

$\eta_{\mathrm{t}, z}^{\mathrm{SOCA}}=\delta v_{z}^{2} \tau_{\mathrm{corr}}$,

we obtained $\eta_{\mathrm{t}, z}^{\mathrm{SOCA}}=6.8 \times 10^{-6}$ for model $E x-4 E-9-R$, while Table 2 gives $\eta_{\mathrm{t}, z}=1.05 \times 10^{-5}$ (as compared to $\eta_{\mathrm{t}, x}=1.81 \times$ $10^{-5}$ for model $\left.E y-4 E-9-R\right)$. Although it is less accurate than the estimates we obtained in the case of radial field diffusion, the prediction of the SOCA still gives a reasonable value for the turbulent resistivity.

Finally, because of the lower values of the turbulent resistivity, the final column of Table 2 reports systematically larger values of the turbulent magnetic Prandtl number, ranging from 1.57 for model $E x-4 E-9-R$ to 3.00 for model $E x-4 E-9-R$. As in the case of a vertical field diffusing radially, $P m_{\mathrm{t}}$ is a slowly increasing function of the strength of the turbulence.

\section{Discussion and conclusion}

In this paper, we have presented a set of local numerical simulations aimed at measuring the properties of turbulent diffusion of magnetic field, quantified using an anomalous turbulent resistivity $\eta_{\mathrm{t}}$, resulting from MHD turbulence induced by the MRI. We considered only the case in which turbulence develops in the absence of a mean magnetic field. We used two different codes, Athena and Ramses, and vary both the box size and the magnetic field geometry. The main result that emerges from our simulations is that $\eta_{\mathrm{t}}$ tends to be slightly smaller, but similar in magnitude, to the anomalous viscosity $v_{\mathrm{t}}$ that can be associated with the outward transport of angular momentum due to the turbulence. In other words, their ratio, the turbulent magnetic number $P m_{\mathrm{t}}$, is of order unity (actually in most of our runs it was slightly larger than one). We also found that our results are roughly consistent with the predictions of the mean field theory SOCA. Perhaps more relevant is the fact that our results are consistent with those recently published by Guan \& Gammie (2009) and Lesur \& Longaretti (2009), who used different field topologies (toroidal and vertical mean field), different numerical methods (similar to those in the ZEUS code, and an incompressible pseudo-spectral code respectively) and, as described in the introduction, different approaches to measure the turbulent resistivity. This broad agreement demonstrates that all these results, obtained and published independently, are largely insensitive to the numerical method and the topogoly of the magnetic field. As suggested already in the literature (Parker 1971), a safe first order guess of the magnitude of the turbulent resistivity is therefore $\eta_{\mathrm{t}} \sim v_{\mathrm{t}}$.

A number of other results emerge from the comparison of the suite of simulations we present here with the work of Guan \& Gammie (2009) and Lesur \& Longaretti (2009). First, it is worth noting that our results and those of Lesur \& Longaretti (2009) were obtained including explicit dissipation, while those of Guan \& Gammie (2009) relied only on numerical dissipation. Since all the results are roughly consistent with one another, this is apparently not an issue as far as determining $P m_{\mathrm{t}}$ is concerned. Moreover, Lesur \& Longaretti (2009) consider the case $P m=1$ while we have used $P m=4$. The broad agreement between both studies therefore suggest that $P m_{\mathrm{t}}$ does not strongly depend on microscopic dissipation coefficients. However, it would be premature to draw definite statements here since other differences between both works might mask a possible $\mathrm{Pm}$ dependence in the results. Although very computationally expensive, a systematic study of the sensitivity of our results to the value of $\mathrm{Pm}$ is needed in the future. Second, $P m_{\mathrm{t}}$ appears to be smaller in large boxes. Indeed, we found $P m_{\mathrm{t}}=0.57$ for model $E y-4 E$ 10-A-L4-lx4, while $P m_{\mathrm{t}}$ is found to be larger than one in small boxes having identical parameters. This rather low value, obtained in a large box and for a small forcing EMF, is very close to the values quoted by Guan \& Gammie (2009) for models having the same radial box size and a weak imposed field. In addition, Guan \& Gammie (2009) considered boxes larger than $4 H$ in the radial direction and found this value of $P m_{\mathrm{t}}$ to be independent of box size. By contrast, both our results and those of Lesur \& Longaretti (2009) suggest a value larger than one (or equivalently fairly low values for the turbulent resisitivity) in small boxes, a regime Guan \& Gammie (2009) did not investigate. Taken together, all these results point toward a decrease of $P m_{\mathrm{t}}$ when box size is increased from values of a few to values of roughly one half, with a plateau obtained for boxes larger than $4 H$.

Despite their broad agreement, it is instructive to consider the differences between our results and the aformentionned papers (Guan \& Gammie 2009; Lesur \& Longaretti 2009). One such difference is that, regardless of the model or the box size, we always find that $P m_{\mathrm{t}}$ increases with the amplitude of the forcing EMF. Lesur \& Longaretti (2009) also report such a trend for the models they label XXZn, in which they consider the vertical diffusion of a toroidal field, as we do in Sect. 4.3. These trend is not so clear for their other cases. However, most of their runs were obtained in the presence of a net vertical flux. The nature of the turbulence is strongly modified in that case and this is probably not appropriate to draw the comparison with those models any further. By contrast, Guan \& Gammie (2009) report no dependence of $P m_{\mathrm{t}}$ on the amplitude of the magnetic field they impose for models that are similar to ours (i.e. the diffusion of a radially varying vertical field). Even if the different field topology they consider (namely a net toroidal flux) plays a role, the difference with our results can most likely be attributed to the different methods that are being used. While the present paper 
aims to measure a steady state response of the flow to a perturbation that is imposed at all times, Guan \& Gammie (2009) superpose an additional field to an already turbulent flow at $t=0$ and let it decay. Thus it is possible that transients associated with this additional field may complicate the estimate of time averaged values for the transport coefficients ${ }^{3}$. In particular, for large strength of the additional field, the flow may not have enough time to reach fully saturated values of the turbulence before the strength of the imposed field decays significantly. This might lead to an underestimate of the value of $\alpha$ and consequently of the value of $P m_{\mathrm{t}}$, masking the increase of $P m_{\mathrm{t}}$ with forcing that we found.

It is also important to stress that all three approaches share common limitations to their analyses. First and foremost, the analysis is by definition local, whereas the diffusion of magnetic field accross the disk is an intrisically global problem that depends on the large scale properties of the disk such as the radial profiles of the surface density and magnetic flux (Spruit \& Uzdensky 2005). Another related limitation is the neglect of vertical density stratification in the disk. Indeed, all three studies presents numerical simulations performed in "unstratified" shearing boxes, neglecting the vertical component of gravity. In simulations including gas density stratification, it was found that MHD turbulence is suppressed in the low density corona above and below the disk midplane (Stone et al. 1996). It is likely that turbulent diffusion will be greatly reduced at those locations, possibly causing the magnetic flux to remain anchored to the disk corona. This could prevent diffusion that otherwise would have been driven by turbulence in the midplane, or by channel modes that couple the upper layers of the disk with the midplane (Bisnovatyi-Kogan \& Lovelace 2007; Rothstein \& Lovelace 2008; Lovelace et al. 2009). A numerical test of this scenario is beyong the scope of the present paper but should be considered in the future. Finally, let us mention the very recent work of Beckwith et al. (2009). Using global simulations that combine the large scale nature of magnetic field diffusion and the vertical density stratication (but, of course, with a dramatic reduction in the spatial resolution), these authors challenge the concept of turbulent resistivity itself and advocate a completely different scenario to describe magnetic flux evolution in accretion disks. Clearly, despite the agreement with already published calculations, the results presented here should be taken with care when used in the context of astrophysical applications, such as jet launching or magnetic flux distribution in accretion disks.

Acknowledgements. We thank J. Goodman for suggesting the method used in this paper to measure the turbulent resistivity, and G. Lesur and P.-Y. Longaretti for stimulating discussions. S.F. acknowledges the Astronomy Department of Princeton University and the Institute of Advanced Studies for their hospitality during visits that enabled this work to be initiated. This work used the computational facilities supported by the Princeton Institute for Computational Science and Engineering.

\section{References}

Balbus, S., \& Hawley, J. 1991, ApJ, 376, 214

Balbus, S., \& Hawley, J. 1998, Rev. Mod. Phys., 70, 1

Beckwith, K., Hawley, J. F., \& Krolik, J. H. 2009, arXiv e-prints

Bisnovatyi-Kogan, G. S., \& Lovelace, R. V. E. 2007, ApJ, 667, L167

Blandford, R. D., \& Payne, D. G. 1982, MNRAS, 199, 883

Brandenburg, A., \& Subramanian, K. 2005, Phys. Rep., 417, 1

Casse, F., \& Ferreira, J. 2000, A\&A, 353, 1115

Fromang, S., \& Nelson, R. P. 2009, A\&A, 496, 597

Fromang, S., \& Papaloizou, J. 2006, A\&A, 452, 751

Fromang, S., Hennebelle, P., \& Teyssier, R. 2006, A\&A, 457, 371

Fromang, S., Papaloizou, J., Lesur, G., \& Heinemann, T. 2007, A\&A, 476, 1123

Gammie, C. F. 2001, ApJ, 553, 174

Gardiner, T. A., \& Stone, J. M. 2005a, J. Comput. Phys., 205, 509

Gardiner, T. A., \& Stone, J. M. 2005b, in Magnetic Fields in the

Universe: From Laboratory and Stars to Primordial Structures., ed. E. M.

de Gouveia dal Pino, G. Lugones, \& A. Lazarian, AIP Conf. Proc., 784, 475

Gardiner, T. A., \& Stone, J. M. 2008, J. Comput. Phys., 227, 4123

Goldreich, P., \& Lynden-Bell, D. 1965, MNRAS, 130, 125

Guan, X., \& Gammie, C. F. 2009, ApJ, 697, 1901

Guan, X., Gammie, C. F., Simon, J. B., \& Johnson, B. M. 2009, ApJ, 694, 1010

Hawley, J., \& Stone, J. 1995, Comput. Phys. Commun., 89, 127

Hawley, J. F., Gammie, C. F., \& Balbus, S. A. 1995, ApJ, 440, 742

Hawley, J. F., Gammie, C. F., \& Balbus, S. A. 1996, ApJ, 464, 690

Heinemann, T., \& Papaloizou, J. C. B. 2008a, MNRAS, 397, 52

Heinemann, T., \& Papaloizou, J. C. B. 2008b, MNRAS, 397, 64

Johansen, A., Youdin, A., \& Klahr, H. 2009, ApJ, 697, 1269

Johnson, B. M., \& Gammie, C. F. 2005, ApJ, 635, 149

Johnson, B. M., Guan, X., \& Gammie, C. F. 2008, ApJS, 177, 373

Landau, L. D., \& Lifshitz, E. M. 1959, Fluid mechanics, Course of theoretical physics (Oxford: Pergamon Press)

Latter, H. N., Lesaffre, P., \& Balbus, S. A. 2009, MNRAS, 394, 715

Lesur, G., \& Longaretti, P.-Y. 2007, MNRAS, 378, 1471

Lesur, G., \& Longaretti, P.-Y. 2009, A\&A, 504, 309

Lovelace, R. V. E., Bisnovatyi-Kogan, G. S., \& Rothstein, D. M. 2009, Nonlinear Processes in Geophys., 16, 77

Lubow, S. H., Papaloizou, J. C. B., \& Pringle, J. E. 1994, MNRAS, 267, 235

Masset, F. 2000, A\&AS, 141, 165

Parker, E. N. 1971, ApJ, 163, 279

Pessah, M. E., \& Goodman, J. 2009, ApJ, 698, 72

Pudritz, R. E., Ouyed, R., Fendt, C., \& Brandenburg, A. 2007, in Protostars and Planets V, ed. B. Reipurth, D. Jewitt, \& K. Keil, 277

Rädler, K.-H., \& Rheinhardt, M. 2007, Geophys. Astrophys. Fluid Dyn., 101, 117

Rothstein, D. M., \& Lovelace, R. V. E. 2008, ApJ, 677, 1221

Schekochihin, A. A., Iskakov, A. B., Cowley, S. C., et al. 2007, New J. Phys., 9, 300

Shakura, N. I., \& Sunyaev, R. A. 1973, A\&A, 24, 337

Simon, J. B., Hawley, J. F., \& Beckwith, K. 2009, ApJ, 690, 974

Spruit, H. C., \& Uzdensky, D. A. 2005, ApJ, 629, 960

Stone, J. M., \& Norman, M. L. 1992, ApJS, 80, 791

Stone, J. M., \& Gardiner, T. A. 2009, ApJ, in preparation

Stone, J. M., Hawley, J. F., Gammie, C. F., \& Balbus, S. A. 1996, ApJ, 463, 656

Stone, J. M., Gardiner, T. A., Teuben, P., Hawley, J. F., \& Simon, J. B. 2008, ApJS, 178, 137

Teyssier, R. 2002, A\&A, 385, 337

van Ballegooijen, A. A. 1989, in Accretion Disks and Magnetic Fields in Astrophysics, ed. G. Belvedere, Astrophys. Space Sci. Library, 156, 99

\footnotetext{
3 Of course, such transients are most likely of physical origin, in the sense that the decay timescale measured by Guan \& Gammie (2009) is related to the relaxation timescale of the turbulence. Nevertheless, their presence complicate the estimate of $P m_{\mathrm{t}}$
} 